\title{
Economic Evaluation of Warfarin, Dabigatran, Rivaroxaban, and Apixaban for Stroke Prevention in Atrial Fibrillation
}

\author{
Torbjørn Wisløff • Gunhild Hagen • \\ Marianne Klemp
}

Published online: 9 April 2014

(c) The Author(s) 2014. This article is published with open access at Springerlink.com

\begin{abstract}
Background Atrial fibrillation is a major risk factor for stroke, which causes thousands of deaths and sequelae. It is recommended that atrial fibrillation patients at medium or high risk of stroke use an oral anticoagulant to reduce the risk of stroke. In the past few years, three new oral anticoagulants (NOACs), dabigatran, rivaroxaban, and apixaban, have been introduced in competition to the older oral anticoagulant warfarin.

Objective The objective of this study was to evaluate the relative cost effectiveness of warfarin, dabigatran, rivaroxaban, and apixaban in a Norwegian setting.

Methods We created a probabilistic decision-analytic Markov model to simulate the life of patients with atrial fibrillation. We performed several scenario analyses,
\end{abstract}

Electronic supplementary material The online version of this article (doi:10.1007/s40273-014-0152-z) contains supplementary material, which is available to authorized users.

T. Wisløff $(\bowtie) \cdot$ G. Hagen · M. Klemp

Norwegian Knowledge Centre for the Health Services, Postboks 7004, St. Olavs Plass, 0130 Oslo, Norway

e-mail: twisloff@gmail.com

T. Wisløff

Department of Biostatistics, Epidemiology and Health

Economics, Oslo University Hospital, Oslo, Norway

T. Wisløff

Department of Health Management and Health Economics, University of Oslo, Oslo, Norway

G. Hagen

Department of Public Health and General Practice, Norwegian

University of Science and Technology, Trondheim, Norway

M. Klemp

Department of Pharmacology, University of Oslo, Oslo, Norway including changing the switching age for dabigatran from 80 to 75 years old.

Results Assuming the European Society of Cardiology guidance, sequential dabigatran $(2 \times 150 \mathrm{mg}$ daily until 80 years old, $2 \times 110 \mathrm{mg}$ thereafter) seems to be the most cost-effective alternative for high-risk AF patients. For medium-risk patients, apixaban $(2 \times 5 \mathrm{mg}$ daily $)$ seems to be somewhat more effective than dabigatran, but dabigatran is still marginally the most cost-effective alternative. In scenario analyses reducing dabigatran from $2 \times 150 \mathrm{mg}$ to $2 \times 110 \mathrm{mg}$ at the age of 75 years (instead of at age 80), apixaban $(2 \times 5 \mathrm{mg}$ daily $)$ becomes the most cost-effective alternative for both risk groups.

Conclusion We have found apixaban or sequential dabigatran to be the alternatives most likely to be considered cost effective, depending on the switching age for dabigatran. These conclusions are highly sensitive to assumptions made in the analysis. 


\section{Key Points for Decision Makers}

The new oral anticoagulants, dabigatran, rivaroxaban, and apixaban, are likely to yield additional health benefits in terms of quality-adjusted life-years as compared with warfarin.

Differences in health gains are, however, relatively small and prices are high.

Sequential dabigatran $(150 \mathrm{mg}$ up to age 80 years, thereafter $110 \mathrm{mg}$ as recommended by the European Society of Cardiology) is the strategy most likely to be considered cost effective, regardless of risk group.

When reducing the dabigatran dosage at age 75 years (instead of at age 80 years), apixaban becomes the most effective and cost-effective alternative.

Conclusions are highly dependent upon assumptions made in the analysis.

\section{Introduction}

Atrial fibrillation (AF) is a common heart disease comprising irregular heartbeat and is a major risk factor for stroke [1]. To reduce the risk of stroke, for decades AF patients have been treated with the oral anticoagulant warfarin. However, warfarin leads to an increased risk of bleeding, and requires close monitoring and follow-up from medical doctors. The trade-off between decreased risk of stroke and increased risk of bleeding may be difficult for both doctors and patients because the recommended dosage of warfarin is highly individual.

As the average age in most countries is increasing, so is the prevalence of AF, now reported to be between 1.5 and $2 \%$ in developed countries [2]. The prevalence of AF in the last US stroke statistics is reported to be between 0.5 and $8.8 \%$ for different age groups [3]. Likewise, more than $70 \%$ of AF patients in the USA were reported to use anticoagulants [3], with somewhat smaller numbers in Europe [4].

Until recently, guidelines have recommended the use of warfarin for AF patients with two additional risk factors for stroke [5]. With the introduction of new oral anticoagulants (NOACs), guidelines are less strict; oral anticoagulants are now recommended for AF patients with only one additional risk factor $[6,7]$.

The increased prevalence of AF, combined with more patients being eligible for treatment, makes it increasingly important to explore whether the benefits of NOACs outweigh the downsides with regards to health. And if the health effects are positive, is the increased effectiveness large enough to justify the added cost? Secondly, in the case that NOACs could be considered cost-effective alternatives to warfarin, it is of interest which of the three would yield the most value for the money. In the first 4 years after the introduction of dabigatran (the first NOAC), several economic evaluations have compared NOACs with warfarin [8]. However, most of these only compared one NOAC with warfarin. This in not in concordance with recently published guidelines for health economic evaluations, where analysts are recommended to include all feasible interventions in the analyses [9]. To date, only two published analyses have compared all oral anticoagulants in one analysis $[10,11]$. Both of these analyses were performed before the price of apixaban was available in the relevant countries and the analyses are therefore based on an assumed price, which is obviously an important limitation, as conclusions on cost effectiveness are highly price dependent.

Our objective in this article was to first develop a model that simulates what happens to AF patients taking warfarin or the NOACs, and second to estimate the cost effectiveness of the NOACs compared with warfarin and each other. The perspective of the analysis is the Norwegian publicly financed healthcare system.

\section{Methods}

In order to evaluate the relative cost effectiveness of dabigatran, rivaroxaban, apixaban, and warfarin, we created a probabilistic decision-analytic model, the NOrwegian STRoke and Atrial fibrillation model (NOSTRA). NOSTRA was created as a Markov model, built around clinical events and health states. A Markov structure was chosen in order to be able to capture long-term health and cost consequences of the different treatment options. A simplified version of the model that illustrates all events in the first cycles is depicted in Fig. 1, while the whole structure, as created in TreeAgePro ${ }^{\circledR}$, is available in Appendix 1 of the Electronic Supplementary Material.

In addition to the health state "AF without further complications," five health outcomes with considerable potential impact on patients were included in the NOSTRA model: ischemic stroke (IS), intracranial bleeding (IB), major gastrointestinal bleeding (MGIB), acute myocardial infarction (AMI), and death (Fig. 1). Risks of events are incorporated into the model as 1-year probabilities adjustable for different risk factors according to $\mathrm{CHA}_{2} \mathrm{DS}_{2}-\mathrm{VASc}$ and HAS-BLED [12, 13]. $\mathrm{CHA}_{2} \mathrm{DS}_{2}$-VASc and HASBLED are scoring algorithms that divide patients into risk 
Fig. 1 Model structure (arrows represent health events, while boxes are health states). $A F$ atrial fibrillation, $A M I$ acute myocardial infarction, $G I$ gastrointestinal, intra. intracranial, isch. ischemic, mod. moderate, prev. previous, seq. sequelae, sev. severe

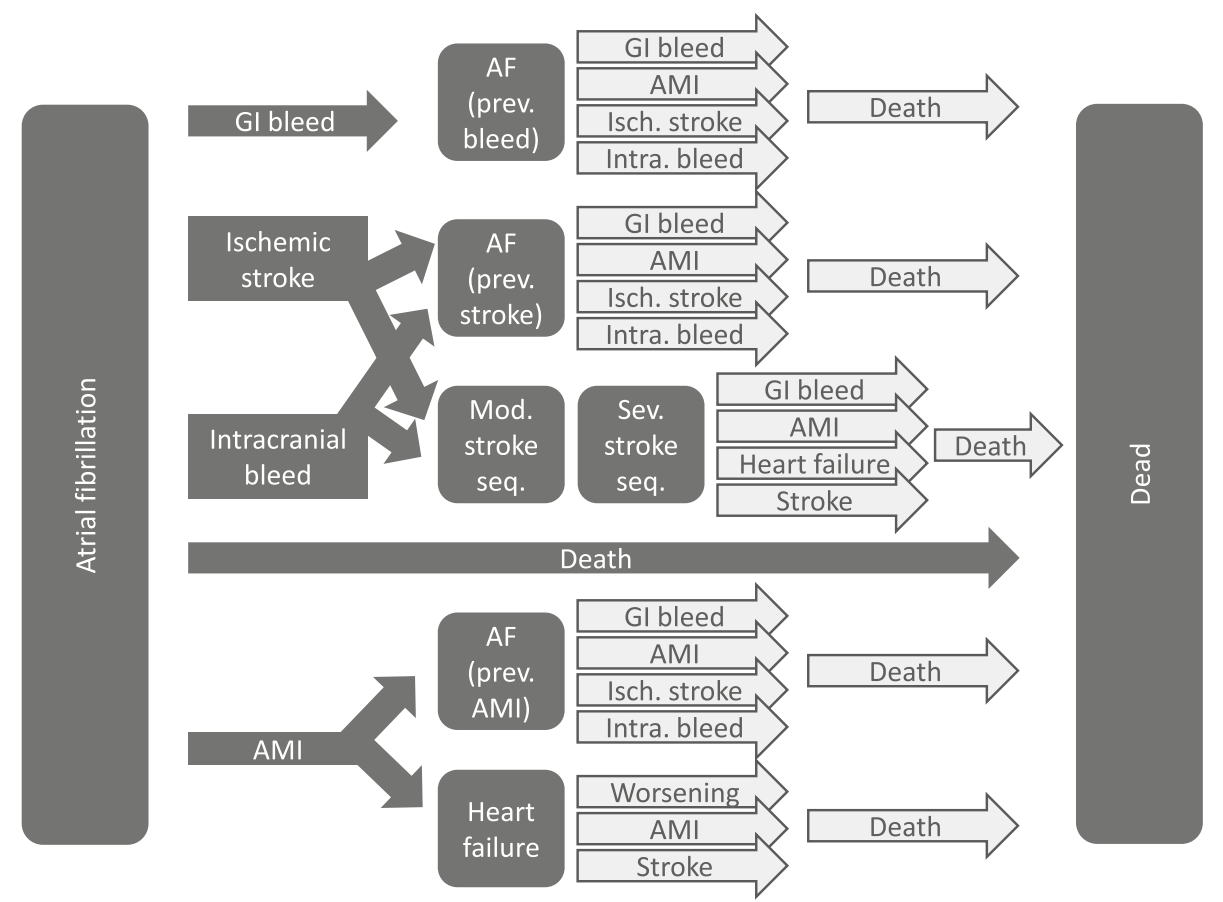

groups according to how many of a predefined set of clinical risk factors they have. For $\mathrm{CHA}_{2} \mathrm{DS}_{2}-\mathrm{VASc}$, these are Congestive heart failure, Hypertension, Age $\geq 75$ years, Diabetes mellitus, Prior stroke (or transient ischemic attack, or thromboembolism), Vascular disease, Age 65-74 years, and Sex (female). For HAS-BLED, the risk factors are Hypertension, Abnormal renal or liver function, Stroke, Bleeding, Labile international normalized ratio (INR), Elderly (age > 65 years), and Drugs or alcohol.

After each event, patients are either successfully treated for their condition and assumed to be more or less asymptomatic, or they are assumed to have developed heart failure or stroke sequelae. The dark arrows in Fig. 1 illustrate the possible events in the first cycle of the model, while the white arrows illustrate the possible events in later cycles. The final outcome of the model is death, and simulations were stopped at the stage where all patients entering the model would have been 105 years old; hence, a lifetime time horizon was applied.

Risks of baseline clinical events were in the model mainly based on Scandinavian registry data [14-24], and to some extent on international data [25-29] as reported in Table 1. Risks of most primary events (IB, MGIB, AMI, and death) were based on a population with AF patients all using warfarin. Risk of IS was based on a registry of AF patients who did not use warfarin. This risk was adjusted down based on other registry data to give a better representation of the risk for warfarin patients [30]. The proportion of stroke patients that have severe $(20 \%)$ and moderate $(48 \%)$ sequelae after 1 year was based on Swedish registry data. Age-specific mortality rates from the general Norwegian population were multiplied by hazard ratios estimated in a Swedish population [31] to give estimated mortality among AF patients in Norway.

To model what happens to patients if they are started on NOACs instead of warfarin, rates of events were multiplied by hazard ratios of these events based on data from randomized clinical trials (Table 2). Efficacy data were based on intention-to-treat analyses from the three major randomized clinical trials comparing each of the NOACs with warfarin [32-34]. We used overall efficacy data for all different subgroups of $\mathrm{CHA}_{2} \mathrm{DS}_{2}$-VASc and HAS-BLED, since the overall estimate of efficacy can also be considered a reasonable estimate for subgroups [35].

Due to an increased risk of bleeding with dabigatran $150 \mathrm{mg}$, guidelines from the European Society of Cardiology (ESC) recommend taking dabigatran $2 \times 110 \mathrm{mg}$ and not $2 \times 150 \mathrm{mg}$ above the age of 80 years. In the European public assessment report (EPAR) from the European Medicines Agency (EMA), the age 75 years is suggested for some groups [36]. In our analyses, we assumed that patients who started on dabigatran $(2 \times 150 \mathrm{mg})$ before age 80 years switched to $2 \times 110 \mathrm{mg}$ on their 80 th birthday (this scenario is from this point onwards called sequential dabigatran). In separate scenario analyses, we explored to what extent this assumption affected the results, when analyzing a scenario where the switch is performed at age 75 years.

All costs and prices used in the model were from 2012, except for the evaluated drugs, because apixaban did not have a price available until 2013. Prices of warfarin, dabigatran, rivaroxaban, and apixaban were gathered from 
Table 1 Key parameters

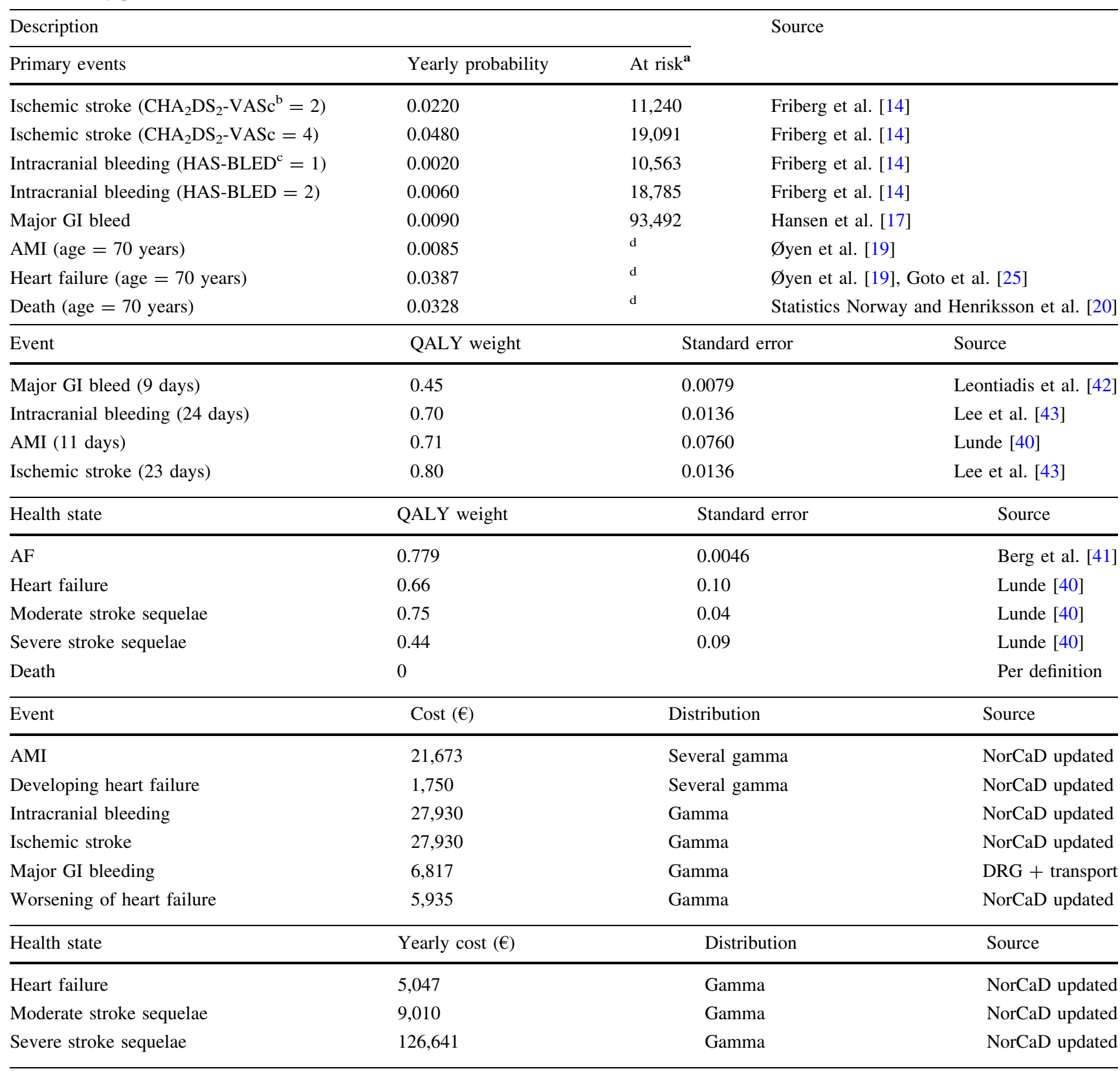

$A F$ atrial fibrillation, $A M I$ acute myocardial infarction, $G I$ gastrointestinal, $Q A L Y$ quality-adjusted life-year, NorCaD Norwegian Cardiovascular disease model [38]

${ }^{a}$ Number at risk used for creating probability distribution (beta). For ischemic stroke and intracranial bleeding, this was based on the Swedish atrial fibrillation cohort study [14], while for major GI bleeding, these were based on a Danish study of the entire population [17]

${ }^{\mathrm{b}} \mathrm{CHA}_{2} \mathrm{DS}_{2}$-VASc $=$ Congestive heart failure, Hypertension, Age $\geq 75$ years, Diabetes mellitus, Prior stroke (or transient ischemic attack, or thromboembolism), Vascular disease, Age 65-74 years, and Sex (female)

${ }^{\mathrm{c}}$ HAS-BLED = Hypertension, Abnormal renal or liver function, Stroke, Bleeding, Labile international normalized ratio, Elderly (age $>65$ years), and Drugs or alcohol

${ }^{\mathrm{d}}$ Risk of AMI, heart failure, and death is based on Norwegian data for patients without AF multiplied by an increased risk due to AF. These data are dependent upon age, but only given here for age 70 years (more details on estimation can be found in Wisløff et al. [58]). The multiplier for AMI is 1.23 based on a large international registry [25], while the multipliers for death decreases from 3.14 to 1.71 depending on age as reported by Swedish data [20]. The multiplier for heart failure is 3.04 based on an international registry [25] 
Table 2 Efficacy from included trials as reported in the Health Technology Assessment report [58] (incorporated as log-normal distributions)

\begin{tabular}{|c|c|c|c|c|c|c|c|c|c|c|c|c|}
\hline & \multicolumn{3}{|c|}{$\begin{array}{l}\text { Apixaban vs. } \\
\text { warfarin }\end{array}$} & \multicolumn{3}{|c|}{$\begin{array}{l}\text { Dabigatran } 110 \text { mg vs. } \\
\text { warfarin }\end{array}$} & \multicolumn{3}{|c|}{$\begin{array}{l}\text { Dabigatran } 150 \mathrm{mg} \text { vs. } \\
\text { warfarin }\end{array}$} & \multicolumn{3}{|c|}{$\begin{array}{l}\text { Rivaroxaban vs. } \\
\text { warfarin }\end{array}$} \\
\hline & HR & $\ln (\mathrm{RR})$ & SE & HR & $\ln (\mathrm{RR})$ & SE & HR & $\ln (\mathrm{RR})$ & SE & HR & $\ln (\mathrm{RR})$ & SE \\
\hline All-cause mortality & 0.89 & -0.12 & 0.06 & 0.91 & -0.09 & 0.06 & 0.88 & -0.13 & 0.07 & 0.92 & -0.08 & 0.06 \\
\hline Ischemic or uncertain stroke & 0.92 & -0.08 & 0.11 & 1.11 & 0.10 & 0.12 & 0.76 & -0.27 & 0.13 & 0.94 & -0.06 & 0.11 \\
\hline Intracranial bleeding & 0.42 & -0.87 & 0.17 & 0.31 & -1.17 & 0.22 & 0.40 & -0.92 & 0.20 & 0.67 & -0.40 & 0.17 \\
\hline Acute myocardial infarction & 0.88 & -0.13 & 0.15 & 1.35 & 0.30 & 0.16 & 1.38 & 0.32 & 0.16 & 0.81 & -0.21 & 0.13 \\
\hline Major gastrointestinal bleeding & 0.89 & -0.12 & 0.12 & 1.10 & 0.10 & 0.13 & 1.50 & 0.41 & 0.12 & 1.60 & 0.47 & 0.11 \\
\hline
\end{tabular}

$H R$ hazard ratio, $\ln (R R)$ natural logarithm (relative risk), $S E$ standard error

Table 3 Prices $(€)$ of investigated drugs

\begin{tabular}{|c|c|c|c|c|c|c|c|}
\hline Interventions & Pills per day & Dosage (mg) & Price & Pills per package & Price per pill & Price per day & Price per year \\
\hline Apixaban & 2 & 5 & 288 & 168 & 1.71 & 3.42 & 1,250 \\
\hline Dabigatran & 2 & 110 & 101 & 60 & 1.68 & 3.36 & 1,228 \\
\hline Dabigatran & 2 & 150 & 101 & 60 & 1.68 & 3.36 & 1,228 \\
\hline Rivaroxaban & 1 & 20 & 292 & 100 & 2.92 & 2.92 & 1,066 \\
\hline Warfarin & 2 & 2.5 & 17 & 100 & 0.17 & 0.33 & 121 \\
\hline
\end{tabular}

the Norwegian Medicines Agency on 19 February 2013 (Table 3). Costs of monitoring related to warfarin treatment were based on cost estimates used in a Norwegian Single Technology Appraisal (STA) of dabigatran [37]. These mean yearly warfarin monitoring costs were assumed to be $€ 640[€ 1=7.47$ Norwegian kroner (NOK)]. Costs of events and health states were, to a large extent, based on data gathered for the Norwegian Cardiovascular Disease (NorCaD) model [38]. These were updated based on Norwegian fees and schedules from 2012 (see Appendix 2 in the Electronic Supplementary Material). Costs and effects were all discounted at $4 \%$ according to Norwegian guidelines for economic evaluations of healthcare interventions [39]. We assumed that incremental effects in terms of quality-adjusted life-years (QALYs) were valued at a cost-effectiveness threshold of $€ 79,000$, due to a suggested threshold for Norway of NOK588,000 per QALY [39].

QALY weights were incorporated into the model for each event and health state [40-44]. QALYs in health states are assumed to last for whole years, while weights for events are only for shorter periods (Table 1). All QALY weights in the model are based on EQ-5D-3L (EuroQol 5 Dimensions 3 Levels), which is the most commonly used instrument for eliciting QALY values [45, 46].

The NOSTRA model is a so-called probabilistic model. This means that all parameters that are considered to be uncertain are included in the model as probability distributions rather than fixed values. Choices of probability distributions were based on common conventions, as described by, for instance, Briggs et al. [47]. The choice of log-normal distribution for efficacy inputs is based on the argument that the standard error for relative risks is defined on the $\log$ scale, as, for instance, also pointed out by Altman [48]. Cost data are usually right skewed, and have been shown to fit well with gamma distributions [49]; hence, we used gamma distributions for the cost input into the model. The relationship between binomial and beta distributions, and the fact that beta distributions can be bounded by 0 and 1 , makes these the obvious choice for probability data [50]. Regarding distribution type for QALY input, we chose to use beta distributions, which have been reported as a reasonable choice when data were not close to zero [50]. In total, 204 different probability distributions were included; more detail can be found in Tables 1 and 2, or in Appendix 3 in the Electronic Supplementary Material. Evaluation of the model was done through simulation; in 10,000 iterations, we drew a random number from each of the 204 probability distributions. For each iteration, we recalculated the expected cost and health outcomes in terms of QALY of the four drugs. Analyses were presented both in classical incremental analyses with incremental cost-effectiveness ratios (ICERs), and also as net health benefit (NHB) analyses. Results are additionally presented as cost-effectiveness acceptability curves (CEACs) and frontiers (CEAFs).

Primary analyses were performed for a hypothetical group of 75-year-old AF patients with either medium or high risk of stroke and bleeding. The medium-risk group was defined as having no clinical risk factors apart from 
their age; hence, a $\mathrm{CHA}_{2} \mathrm{DS}_{2}-\mathrm{VASc}$ of 2 and HAS-BLED of 1. The high-risk group was defined at $\mathrm{CHA}_{2} \mathrm{DS}_{2}-\mathrm{VASc}=4$ and $\mathrm{HAS}-\mathrm{BLED}=2$, which is approximately the risk profile of an average AF patient in Scandinavia [15].

We also performed some additional scenario analyses to test certain assumptions in the model. Firstly, we tested whether conclusions would change if we assumed only 4 years of treatment instead of life-long treatment, which was our base-case assumption.

Secondly, it has been hypothesized that follow-up of AF patients on NOACs will not be as comprehensive in practice as in the clinical trials and that this will result in the real-life effectiveness of the NOACs being somewhat smaller than has been reported in the trials. To explore this, we repeated our analyses with a decline in effectiveness of NOACs compared with warfarin. The effectiveness was decreased by $6 \%$ per quarter, based on the analyses from the EuroHeart survey [51], which indicates that this was the case for warfarin. All three trials of the NOACs report similar discontinuation rates for warfarin and the NOACs; hence, the reported adherence with warfarin may be the best available data until we have more experience with the NOACs.

Seeing that dabigatran $150 \mathrm{mg}$ is not recommended for the elderly due to a high risk of bleeding, our original analyses were based on the assumption that all patients on dabigatran $150 \mathrm{mg}$ switched to $110 \mathrm{mg}$ at the age of 80 years, as suggested by the EMA in the EPAR. However, ESC has recommended switching from 150 to $110 \mathrm{mg}$ at age 75 years. Because our analyses start with 75-year-old patients, this scenario implies only taking the strategy "sequential dabigatran" out of the analyses.

\section{Results}

We estimated that 75-year-old AF patients with a medium risk of stroke according to $\mathrm{CHA}_{2} \mathrm{DS}_{2}-\mathrm{VASc}$ have on average 7.15 remaining QALYs if treated with warfarin (discounted 5.706) (Table 4). The expected increased effectiveness of using NOACs instead of warfarin was 0.100 QALYs for dabigatran $(2 \times 110 \mathrm{mg}), 0.104$ QALYs for rivaroxaban $(1 \times 20 \mathrm{mg}), 0.146 \mathrm{QALYs}$ for sequential dabigatran, and 0.153 QALYs for apixaban $(2 \times 5 \mathrm{mg})$. All NOACs increased lifetime costs compared with warfarin, despite the fact that a considerable reduction in doctor visits and INR testing was included.

The ICER compared with warfarin was $€ 66,000$ per QALY gained for dabigatran $110 \mathrm{mg}$ and $€ 30,000$ for rivaroxaban, indicating both as cost effective at a costeffectiveness threshold of $€ 79,000$. However, sequential dabigatran had both higher expected QALY gain and lower expected costs than both dabigatran $110 \mathrm{mg}$ and rivaroxaban. Hence, sequential dabigatran dominates the other two. When comparing apixaban to sequential dabigatran, the QALY gain of 0.007 gives an ICER of $€ 80,000$; hence, apixaban is marginally not cost effective compared with sequential dabigatran. Seeing that conclusions on cost effectiveness depend heavily on the assumed cost-effectiveness threshold, we performed analyses with varying thresholds (Fig. 2). Based on our analyses, the optimal oral anticoagulant in terms of cost effectiveness is warfarin for thresholds below $€ 16,000$ and sequential dabigatran for thresholds between $€ 16,000$ and $€ 80,000$. For thresholds above $€ 80,000$, apixaban is the most cost effective.

We estimated AF patients with a high risk of stroke according to $\mathrm{CHA}_{2} \mathrm{DS}_{2}$-VASc to have on average 5.79 remaining QALYs left if treated with warfarin (discounted 4.771) (Table 5). The expected increased effectiveness of using NOACs instead was 0.119 QALYs for dabigatran $(2 \times 110 \mathrm{mg}), 0.117 \mathrm{QALYs}$ for rivaroxaban $(1 \times 20 \mathrm{mg})$, 0.175 QALYs for apixaban $(2 \times 5 \mathrm{mg})$, and 0.183 QALYs for sequential dabigatran. As for medium-risk patients, dabigatran $(2 \times 110 \mathrm{mg})$ and rivaroxaban $(1 \times 20 \mathrm{mg})$ increased lifetime costs compared with warfarin; however,

Table 4 Expected lifetime results for medium-risk patients

\begin{tabular}{|c|c|c|c|c|c|c|c|c|c|}
\hline \multirow[t]{2}{*}{ Strategy } & \multirow{2}{*}{$\begin{array}{l}\text { Lifetime } \\
\text { costs }(€)\end{array}$} & \multirow{2}{*}{$\begin{array}{l}\text { Lifetime } \\
\text { QALY }\end{array}$} & \multirow{2}{*}{$\begin{array}{l}\text { Net health } \\
\text { benefit }^{\mathrm{a}}\end{array}$} & \multicolumn{3}{|c|}{ Incremental analysis } & \multicolumn{3}{|l|}{ Vs. warfarin } \\
\hline & & & & $\begin{array}{l}\text { Incremental } \\
\text { costs }(€)\end{array}$ & $\begin{array}{l}\text { Incremental } \\
\text { effects (QALY) }\end{array}$ & $\begin{array}{l}\text { ICER }(€ / \\
\text { QALY) }\end{array}$ & $\begin{array}{l}\text { Incremental } \\
\text { costs }(€)\end{array}$ & $\begin{array}{l}\text { Incremental } \\
\text { effects (QALY) }\end{array}$ & $\begin{array}{l}\text { ICER }(€ / \\
\text { QALY) }\end{array}$ \\
\hline Warfarin & 47,498 & 5.706 & 5.103 & & & & & & \\
\hline $\begin{array}{l}\text { Sequential } \\
\text { dabigatran }\end{array}$ & 49,821 & 5.852 & 5.219 & 2,323 & 0.146 & 15,920 & 2,323 & 0.146 & 15,920 \\
\hline Apixaban & 50,402 & 5.859 & 5.219 & 581 & 0.007 & 79,526 & 2,904 & 0.153 & 18,955 \\
\hline Rivaroxaban & 50,611 & 5.810 & 5.167 & 790 & -0.042 & Dominated & 3,113 & 0.104 & 29,990 \\
\hline $\begin{array}{l}\text { Dabigatran } \\
110 \mathrm{mg}\end{array}$ & 54,104 & 5.806 & 5.119 & 4,283 & -0.046 & Dominated & 6,606 & 0.100 & 66,121 \\
\hline
\end{tabular}

ICER incremental cost-effectiveness ratio, $Q A L Y$ quality-adjusted life-year

a In QALYs; assuming a cost-effectiveness threshold of €79,000 per QALY 
Fig. 2 Cost-effectiveness acceptability curve and frontier of medium-risk atrial fibrillation patients. cost eff. cost effective, $Q A L Y$ quality-adjusted life-year

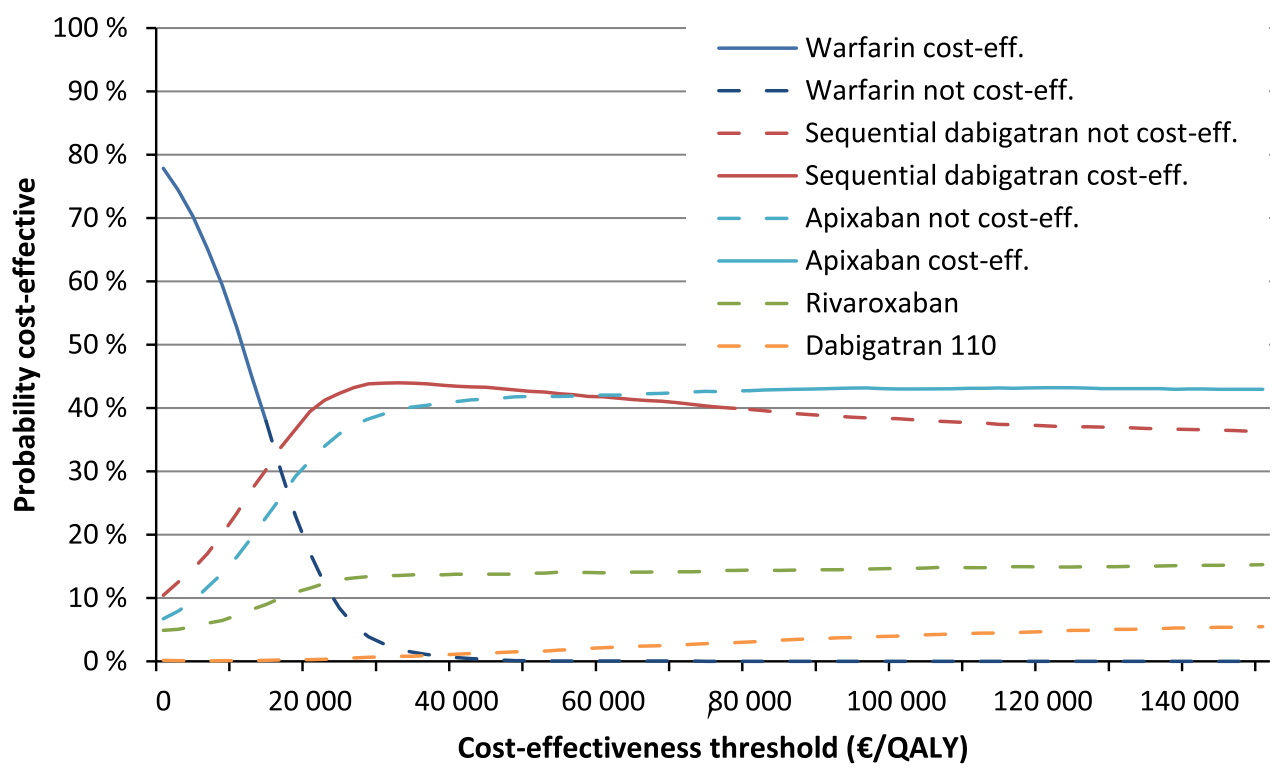

Table 5 Expected lifetime results for high-risk patients

\begin{tabular}{|c|c|c|c|c|c|c|c|c|c|}
\hline \multirow[t]{2}{*}{ Strategy } & \multirow{2}{*}{$\begin{array}{l}\text { Lifetime } \\
\text { costs }(€)\end{array}$} & \multirow{2}{*}{$\begin{array}{l}\text { Lifetime } \\
\text { QALY }\end{array}$} & \multirow{2}{*}{$\begin{array}{l}\text { Net health } \\
\text { benefit }^{\mathrm{a}}\end{array}$} & \multicolumn{3}{|c|}{ Incremental analysis } & \multicolumn{3}{|c|}{ Versus warfarin } \\
\hline & & & & $\begin{array}{l}\text { Incremental } \\
\text { costs }(€)\end{array}$ & $\begin{array}{l}\text { Incremental } \\
\text { effects (QALY) }\end{array}$ & $\begin{array}{l}\text { ICER (€/ } \\
\text { QALY) }\end{array}$ & $\begin{array}{l}\text { Incremental } \\
\text { costs }(€)\end{array}$ & $\begin{array}{l}\text { Incremental } \\
\text { effects (QALY) }\end{array}$ & $\begin{array}{l}\text { ICER }(€ / \\
\text { QALY) }\end{array}$ \\
\hline $\begin{array}{l}\text { Sequential } \\
\text { dabigatran }\end{array}$ & 66,508 & 4.955 & 4.110 & & & & $-2,953$ & 0.183 & $-16,102$ \\
\hline Apixaban & 68,657 & 4.947 & 4.075 & 2,149 & -0.008 & Dominated & -804 & 0.175 & $-4,585$ \\
\hline Warfarin & 69,461 & 4.771 & 3.889 & 2,953 & -0.183 & Dominated & & & \\
\hline Rivaroxaban & 71,849 & 4.888 & 3.975 & 7,402 & -0.064 & Dominated & 2,388 & 0.117 & 20,492 \\
\hline $\begin{array}{l}\text { Dabigatran } \\
110 \mathrm{mg}\end{array}$ & 73,909 & 4.891 & 3.952 & 2,149 & -0.008 & Dominated & 4,448 & 0.119 & 37,250 \\
\hline
\end{tabular}

ICER incremental cost-effectiveness ratio, $Q A L Y$ quality-adjusted life-year

a In QALYs; assuming a cost-effectiveness threshold of $€ 79,000$ per QALY

apixaban $(2 \times 5 \mathrm{mg})$ and sequential dabigatran decreased lifetime costs compared with warfarin (about $-€ 800$ and $-€ 3,000$, respectively). Taking into account that sequential dabigatran both increased effectiveness most and also decreased costs most, this was clearly the more costeffective alternative, a so-called "dominant strategy."

We plotted the CEAC for high-risk AF patients (Fig. 3). We can see that even though the decision seems clear based on the expected values, when taking uncertainty in the parameters into account sequential dabigatran is only $58 \%$ likely to be the cost-effective anticoagulant at the given threshold ( $€ 79,000$ per QALY). On the other hand, sequential dabigatran is the strategy most likely to be cost effective at all cost-effectiveness thresholds.

In scenario analyses we tested whether conclusions would change if we assumed only 4 years of treatment. These analyses indicated that sequential dabigatran was both the most effective and the most cost-effective alternative for both medium-risk and high-risk patients.
In scenario analyses testing a $6 \%$ decrease in NOAC effectiveness per year, the probability of warfarin being cost effective increased from 0.02 to $0.2 \%$ in medium-risk patients, but was unchanged at $0 \%$ in high-risk patients.

In a separate scenario analysis, assuming that patients on dabigatran had to reduce their dose at age 75 , apixaban was the most cost-effective strategy for both risk groups. Accordingly, the percentage of apixaban being the costeffective strategy among all NOACs increased from 43 to $63 \%$ for medium-risk patients and from 35 to $70 \%$ for high-risk patients.

In one-way sensitivity analyses we varied prices of the drugs that were not cost effective at baseline. In mediumrisk patients, apixaban became cost effective at a price reduction of $0.7 \%(€ 1,242$ per annum instead of $€ 1,250$ per annum), while rivaroxaban became cost effective at a reduction of $79 \%$ ( $€ 220$ instead of $€ 1,066$ ). For high-risk patients, a reduction of the apixaban cost per year to $€ 646$ (48\% reduction) made it cost effective compared with 
Fig. 3 Cost-effectiveness acceptability curve and frontier of high-risk atrial fibrillation patients. $Q A L Y$ quality-adjusted life-year

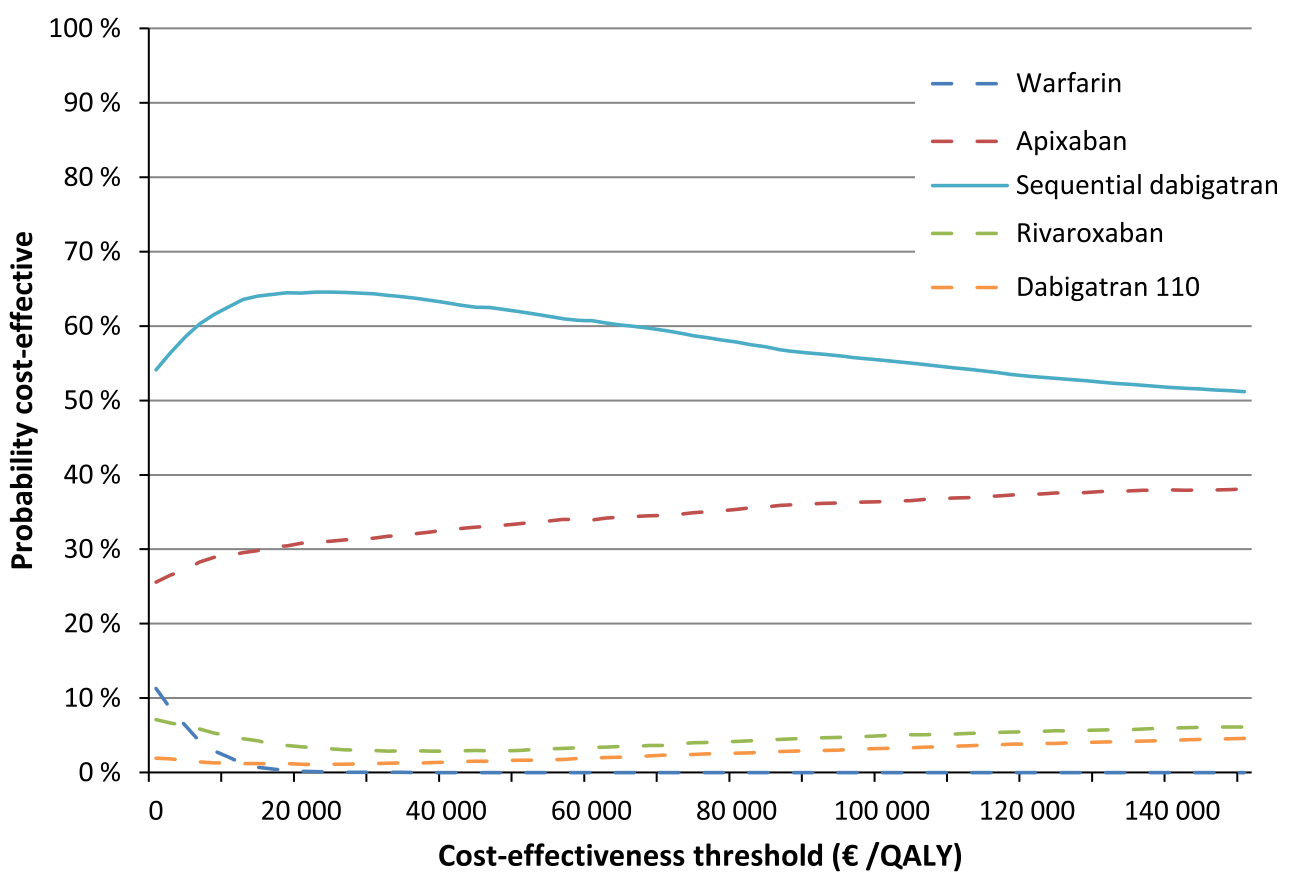

sequential dabigatran. For high-risk patients, no rivaroxaban reduction could make it cost effective, while dabigatran $110 \mathrm{mg}$ and warfarin would never be cost effective in either risk group.

\section{Discussion}

All three NOACs are effective and can be considered cost effective compared with warfarin from a Norwegian perspective. Apixaban is the only NOAC that indicates a positive effect compared with warfarin for all five outcomes included in the NOSTRA model, as can be seen in Table 2 (although only efficacy on IB and mortality are statistically significant at the $5 \%$ level). The increased effectiveness is, however, marginally not cost effective compared with sequential dabigatran, since the ICER was $€ 80,000$ and the proposed cost-effectiveness threshold in Norway is $€ 79,000$.

Note also from Fig. 2 that apixaban has the highest probability of being cost effective at WTPs above $€ 60,000$. The discrepancy between this probability of being cost effective and the expected cost effectiveness has previously been discussed in detail by, for example, Fenwick et al. [52], Claxton et al. [53], and $\mathrm{Al}$ [54]. The point made here is that if one or more input distribution is not symmetrical, then the intervention with the highest expected net benefit is not necessarily the intervention that is most likely to be cost effective.

For AF patients with a high risk of stroke, sequential dabigatran is both the most effective and most cost effective, despite the fact that dabigatran $150 \mathrm{mg}$ is related to an increased risk of gastrointestinal bleeding and myocardial infarction as compared with warfarin (and indirectly apixaban, as can be inferred from Table 2). This result comes as a consequence of dabigatran having a somewhat better effectiveness than apixaban and warfarin on both IS and IB in the clinical trials.

There is considerable uncertainty related to which of the three oral anticoagulants is the most effective and cost effective for patients with AF. As shown both in the probabilistic analyses and in the scenario analyses, small changes in assumptions could easily change conclusions, not only regarding which NOAC, but also regarding warfarin. The two strategies with the highest probabilities of being cost effective are clearly sequential dabigatran and apixaban in both scenarios. For medium-risk patients, the cost effectiveness seems undecided between the two, since the ICER is so close to the Norwegian suggested WTP, which is still only a reference value. For patients with a higher risk of stroke, the negative effects of dabigatran $150 \mathrm{mg}$ are overshadowed by the positive and sequential dabigatran has the highest probability of being cost effective.

Needless to say, the prices of the NOACs affect the results. This is especially so for medium-risk patients, where a price reduction of less than $1 \%$ for apixaban makes it cost effective compared with sequential dabigatran. Note also that for medium-risk patients, warfarin is the most costeffective alternative when the cost-effectiveness threshold is low. Hence, if the Norwegian threshold is lowered, warfarin may be a cost-effective alternative. 
As mentioned in the Introduction, we have only been able to find two economic evaluations comparing all NOACs in one analysis, as we do here. The results of these analyses were close to ours, in that apixaban and dabigatran $(150 \mathrm{mg})$ were both cost effective compared with warfarin in the base-case, with conclusions favoring apixaban in one study and dabigatran in the other $[10,11]$. The models used in these two analyses seem to be relatively similar to ours; the number of health states, age groups analyzed, follow-up time, discounting, and several other factors were similar. However, a few differences between our model and these two analyses are present. Both of the two other models include more health events than ours, such as minor bleeding. This may be a limitation with our model, but as long as it is minor bleedings, both the QALY impact and cost impact of these are seemingly small. One advantage of our model is that it facilitates analyses with the new risk-scoring system $\mathrm{CHA}_{2} \mathrm{DS}_{2}-\mathrm{VASc}$, which is, for instance, recommended by the ESC [2]. Note also that QALY gains from our model are very close to those reported by Coyle et al. [10], while Harrington et al. [11] report much higher incremental gains.

In the present study, we have presented graphs of CEAC and CEAF in the same figure (Figs. 2 and 3). This was an attempt to join the advantages of the two into one figure. The CEAC gives information of the probability of each intervention being the most cost effective, while the CEAF only gives this information for the strategy with the highest expected net benefit.

There are several limitations in this economic evaluation. The most obvious may be that in modeling some simplifications have to be made [55]. In the NOSTRA model, we have only included some of the events and health states that could potentially be included. We have, however, included the most severe and prevalent, so the model should be thorough enough to have captured most of the relevant differences between the NOACs and warfarin. Additional health outcomes, which potentially could have been included in NOSTRA, are major bleedings (not gastrointestinal) and hemorrhagic stroke. The first of these was not included because it would require splitting the outcome "major bleedings" from the trials into subgroups of bleedings in order to find proper cost estimates. The second outcome (hemorrhagic stroke) was excluded because of the major overlap with IB, which would imply double counting if it was included. With a total of eight different health states and 204 different probability distributions included, the NOSTRA model is clearly among the more comprehensive models.

A model is, however, never better than its input parameters. Efficacy data for each of the comparisons with warfarin are based only on one single RCT each and direct active comparisons between the NOACs have not yet been studied. To be able to prioritize between the different
NOACs, comparative effectiveness is required, preferably through head-to-head RCTs. Relatively short follow-up times in the included trials are also a limitation, because these are drugs that are intended for use for the remainder of people's lives, and they are only studied for approximately 2-3 years. Even though this may be sufficient with regard to efficacy, several safety issues demand longer follow-up. Another issue with the three trials is that they are designed as non-inferiority trials. When non-inferiority trials indicate superiority over warfarin for some outcomes, this is usually taken to be hypothesis generating. Hence, based on those trials, we have generated a hypothesis that NOACs are better than warfarin. However, this has not yet been tested in a superiority trial, and could therefore be seen as a limitation of our modeling results.

A re-analysis of one of the RCTs on which we have based our efficacy data [56] indicated that the effect of dabigatran versus warfarin is smaller (if any) for the subgroup of patients in whom INR levels are well-controlled. The Nordic countries are among the countries with the best INR control, also exemplified in the re-analysis. However, the re-analysis did indicate that Norway might have the worst INR control within this subgroup. Our cost-utility analysis is based on efficacy data from each study as a whole, and no subgroup analyses of efficacy have been used. If similar subgroup differences are shown for the other NOACs, both efficacy and cost effectiveness would be affected. Hence, it would be relevant to re-do both metaanalyses and cost-utility analyses to see whether the NOACs are still effective and cost effective compared with warfarin in settings with different levels of INR control.

Another issue that may be a limitation in these analyses is that we had no data on patient compliance apart from what is reported in the trials. Patients on warfarin are likely to be followed up more closely than patients on NOACs in general practice. Level of follow-up from doctors is likely to be linked to compliance within patients. In two of the three trials on which we based our efficacy data, patients and doctors were both blinded with regards to treatment. Hence, we could not be sure of the effectiveness of these drugs in real practice.

Valuing health-related quality of life is not straightforward, and the use of QALYs requires caution. In the present article, we decided to base all QALY values on EQ-5D data. EQ-5D is the most used tool and the only tool for which we could find published values on all events and health states for input into our model. QALY values obtained for the different health events and states are based on four different studies (See Table 1). It would, of course, be better if EQ-5D had been reported for all these outcomes in one single study. The fact that these values are based on different sources may have shifted the internal ranking between these and then influenced the cost effectiveness. 
Approximately 65,000 patients in Norway and at least 120,000 in Denmark have AF [15, 57]. Of these, about $10 \%$ have a low risk of stroke and are not eligible for anticoagulant treatment. Another subpopulation is also not eligible for NOACs due to co-morbidity or possible drug interactions. We have no data on this percentage, but as an estimate, let's say this is 10-20\% of AF patients. Based on this, around 70-80 \% of AF patients would be eligible for anticoagulant treatment. With a European population of 750 million, between 11 and 15 million people could be assumed to have AF and, of these, perhaps 10 million are eligible for NOACs. A price increase of close to $€ 1,000$ per year per patient would mean around $€ 10$ billion in extra expenditures each year if NOACs were to be used in all patients instead of warfarin.

\section{Conclusion}

There is considerable uncertainty regarding which of the oral anticoagulants is the most cost-effective alternative. However, apixaban and dabigatran (150 mg up to age 80 years, $110 \mathrm{mg}$ after age 80 years) seem to be the most effective and cost-effective alternatives. Warfarin can only be a cost-effective alternative in Norway if the threshold for cost effectiveness is much lower than assumed.

Acknowledgments We are grateful to Tove Ringerike and Åsmund Reikvam for their work related to data on efficacy and Ingrid Harboe for help with literature searches.

Funding sources No financial support was given to any of the researchers for this work, except salaries from the Norwegian Knowledge Centre for the Health Services.

Disclosures The authors have no conflicts of interest.

Author contributions All authors had substantial contributions in the idea phase and the writing process. TW and GH developed the model. TW is the guarantor for the overall content. All authors have reviewed and agreed the final version of the manuscript.

Open Access This article is distributed under the terms of the Creative Commons Attribution Noncommercial License which permits any noncommercial use, distribution, and reproduction in any medium, provided the original author(s) and the source are credited.

\section{References}

1. Friberg J, Scharling H, Gadsboll N, Truelsen T, Jensen GB. Comparison of the impact of atrial fibrillation on the risk of stroke and cardiovascular death in women versus men (The Copenhagen City Heart Study). Am J Cardiol. 2004;94(7):889-94.

2. Camm AJ, Lip GY, De CR, Savelieva I, Atar D, Hohnloser SH, et al. 2012 focused update of the ESC Guidelines for the management of atrial fibrillation: an update of the 2010 ESC Guidelines for the management of atrial fibrillation-developed with the special contribution of the European Heart Rhythm Association. Europace. 2012;14(10):1385-413.

3. Roger VL, Go AS, Lloyd-Jones DM, Benjamin EJ, Berry JD, Borden WB, et al. Heart disease and stroke statistics-2012 update: a report from the American Heart Association. Circulation. 2012;125(1):e2-220.

4. Rivero-Ayerza M, Scholte Op RW, Lenzen M, Theuns DA, Jordaens L, Komajda M, et al. New-onset atrial fibrillation is an independent predictor of in-hospital mortality in hospitalized heart failure patients: results of the EuroHeart Failure Survey. Eur Heart J. 2008;29(13):1618-24.

5. Singer DE, Albers GW, Dalen JE, Fang MC, Go AS, Halperin JL, et al. Antithrombotic therapy in atrial fibrillation: American College of Chest Physicians evidence-based clinical practice guidelines (8th edition). Chest. 2008;133(6 Suppl):546S-92S.

6. Camm AJ, Kirchhof P, Lip GY, Schotten U, Savelieva I, Ernst S, et al. Guidelines for the management of atrial fibrillation: the Task Force for the Management of Atrial Fibrillation of the European Society of Cardiology (ESC). Eur Heart J. 2010;31(19): 2369-429.

7. You JJ, Singer DE, Howard PA, Lane DA, Eckman MH, Fang $\mathrm{MC}$, et al. Antithrombotic therapy for atrial fibrillation: antithrombotic therapy and prevention of thrombosis, 9th ed: American College of Chest Physicians evidence-based clinical practice guidelines. Chest. 2012;141(2 Suppl):e531S-75S.

8. Limone BL, Baker WL, Kluger J, Coleman CI. Novel anticoagulants for stroke prevention in atrial fibrillation: a systematic review of cost-effectiveness models. PLoS One. 2013;8(4):e62183.

9. Caro JJ, Briggs AH, Siebert U, Kuntz KM. Modeling good research practices-overview: a report of the ISPOR-SMDM Modeling Good Research Practices Task Force-1. Med Decis Making. 2012;32(5):667-77.

10. Coyle D, Coyle K, Cameron C, Lee K, Kelly S, Steiner S, et al. Cost-effectiveness of new oral anticoagulants compared with warfarin in preventing stroke and other cardiovascular events in patients with atrial fibrillation. Value Health. 2013;16(4): 498-506.

11. Harrington AR, Armstrong EP, Nolan PE Jr, Malone DC. Costeffectiveness of apixaban, dabigatran, rivaroxaban, and warfarin for stroke prevention in atrial fibrillation. Stroke. 2013;44(6): 1676-81.

12. Lip GY, Nieuwlaat R, Pisters R, Lane DA, Crijns HJ. Refining clinical risk stratification for predicting stroke and thromboembolism in atrial fibrillation using a novel risk factor-based approach: the Euro Heart Survey on Atrial Fibrillation. Chest. 2010;137(2):263-72.

13. Pisters R, Lane DA, Nieuwlaat R, de Vos CB, Crijns HJ, Lip GY. A novel user-friendly score (HAS-BLED) to assess 1-year risk of major bleeding in patients with atrial fibrillation: the Euro Heart Survey. Chest. 2010;138(5):1093-100.

14. Friberg L, Rosenqvist M, Lip GY. Evaluation of risk stratification schemes for ischaemic stroke and bleeding in 182678 patients with atrial fibrillation: the Swedish atrial fibrillation cohort study. Eur Heart J. 2012;33(12):1500-10.

15. Olesen JB, Lip GY, Hansen ML, Hansen PR, Tolstrup JS, Lindhardsen $\mathrm{J}$, et al. Validation of risk stratification schemes for predicting stroke and thromboembolism in patients with atrial fibrillation: nationwide cohort study. BMJ. 2011;342:d124.

16. Harmsen P, Wilhelmsen L, Jacobsson A. Stroke incidence and mortality rates 1987 to 2006 related to secular trends of cardiovascular risk factors in Gothenburg, Sweden. Stroke. 2009;40(8): 2691-7.

17. Hansen ML, Sorensen R, Clausen MT, Fog-Petersen ML, Raunso J, Gadsboll N, et al. Risk of bleeding with single, dual, or triple 
therapy with warfarin, aspirin, and clopidogrel in patients with atrial fibrillation. Arch Intern Med. 2010;170(16):1433-41.

18. Olesen JB, Lip GY, Lindhardsen J, Lane DA, Ahlehoff O, Hansen ML, et al. Risks of thromboembolism and bleeding with thromboprophylaxis in patients with atrial fibrillation: a net clinical benefit analysis using a 'real world' nationwide cohort study. Thromb Haemost. 2011;106(4):739-49.

19. Øyen N, Nygård O, Igland J, Tell GS, Nordrehaug JE, Irgens LM, et al. Hospital admission rates for cardiovascular diseases in Western Norway, 1992-2001 [in Norwegian]. Tidsskr Nor Laegeforen. 2008;128(1):17-23.

20. Henriksson KM, Farahmand B, Johansson S, Asberg S, Terent A, Edvardsson N. Survival after stroke-the impact of CHADS2 score and atrial fibrillation. Int J Cardiol. 2010;141(1):18-23.

21. Shafazand M, Rosengren A, Lappas G, Swedberg K, Schaufelberger M. Decreasing trends in the incidence of heart failure after acute myocardial infarction from 1993-2004: a study of 175,216 patients with a first acute myocardial infarction in Sweden. Eur J Heart Fail. 2011;13(2):135-41.

22. Tveit A, Flonaes B, Aaser E, Korneliussen K, Froland G, Gullestad L, et al. No impact of atrial fibrillation on mortality risk in optimally treated heart failure patients. Clin Cardiol. 2011;34(9): 537-42.

23. Riks-Stroke. Yearly report 2010 [in Swedish]. http://www.riksstroke.org/content/analyser/Riks-Stroke_\%C3\%85rsrapport2010. pdf (2011). Accessed 22 Mar 2014.

24. Olesen JB, Lip GY, Hansen PR, Lindhardsen J, Ahlehoff O, Andersson $\mathrm{C}$, et al. Bleeding risk in 'real world' patients with atrial fibrillation: comparison of two established bleeding prediction schemes in a nationwide cohort. J Thromb Haemost. 2011;9(8):1460-7.

25. Goto S, Bhatt DL, Rother J, Alberts M, Hill MD, Ikeda Y et al. Prevalence, clinical profile, and cardiovascular outcomes of atrial fibrillation patients with atherothrombosis. Am Heart $\mathbf{J}$ 2008;156(5):855-63, 863.e2.

26. Hillen T, Coshall C, Tilling K, Rudd AG, McGovern R, Wolfe $\mathrm{CD}$. Cause of stroke recurrence is multifactorial: patterns, risk factors, and outcomes of stroke recurrence in the South London Stroke Register. Stroke. 2003;34(6):1457-63.

27. Caro JJ, Migliaccio-Walle K, Ishak KJ, Proskorovsky I, O’Brien JA. The time course of subsequent hospitalizations and associated costs in survivors of an ischemic stroke in Canada. BMC Health Serv Res. 2006;6:99.

28. Mosterd A, Cost B, Hoes AW, de Bruijne MC, Deckers JW, Hofman A, et al. The prognosis of heart failure in the general population: the Rotterdam Study. Eur Heart J. 2001;22(15): 1318-27.

29. Cleland JG, Swedberg K, Follath F, Komajda M, Cohen-Solal A, Aguilar JC, et al. The EuroHeart Failure survey programme-a survey on the quality of care among patients with heart failure in Europe. Part 1: patient characteristics and diagnosis. Eur Heart J. 2003;24(5):442-63.

30. Banerjee A, Lane DA, Torp-Pedersen C, Lip GY. Net clinical benefit of new oral anticoagulants (dabigatran, rivaroxaban, apixaban) versus no treatment in a 'real world' atrial fibrillation population: a modelling analysis based on a nationwide cohort study. Thromb Haemost. 2012;107(3):584-9.

31. Henriksson KM, Andersson T, Magnusson A, Bryngelsson I-L, Frobert O, Edvardsson N, et al. A cohort study of all-cause mortality in all patients with a first time hospitalization with atrial fibrillation, versus matched controls, In Sweden during 15 years, 1995-2009 [conference abstract]. Pharmacoepidemiol Drug Saf. 2011;20:S22-3.

32. Connolly SJ, Ezekowitz MD, Yusuf S, Eikelboom J, Oldgren J, Parekh A, et al. Dabigatran versus warfarin in patients with atrial fibrillation. N Engl J Med. 2009;361(12):1139-51.
33. Patel MR, Mahaffey KW, Garg J, Pan G, Singer DE, Hacke W, et al. Rivaroxaban versus warfarin in nonvalvular atrial fibrillation. N Engl J Med. 2011;365(10):883-91.

34. Granger CB, Alexander JH, McMurray JJ, Lopes RD, Hylek EM, Hanna M, et al. Apixaban versus warfarin in patients with atrial fibrillation. N Engl J Med. 2011;365(11):981-92.

35. Oxman AD. Subgroup analyses. BMJ. 2012;344:e2022.

36. European Medicines Agency. EPAR summary for the public: pradaxa, dabigatran etexilate (EMA/544606/2012). http://www. ema.europa.eu/docs/en_GB/document_library/EPAR_-_Summary_ for_the_public/human/000829/WC500041060.pdf. (2012). Accessed 22 Mar 2014.

37. Norwegian Medicines Agency. Reimbursement report: dabigatran (Pradaxa) for prevention of stroke and systemic embolism [in Norwegian]. http://www.legemiddelverket.no/Blaa_resept_og_ pris/blaaresept_forhaandsgodkjent_refusjon/refusjonsbeslutninger/ Documents/2012-2011/Pradaxa_atrieflimmer_2012.pdf (2012). Accessed 22 Mar 2014.

38. Wisløff T, Selmer R, Halvorsen S, Kristiansen IS. Norwegian Cardiovascular Disease Model (NorCaD) - a simulation model for estimating health benefits and cost consequences of cardiovascular interventions. 23. 2008. Report from Kunnskapssenteret (Norwegian Knowledge Centre for the Health Services), No. 23-2008. http://www.kunnskapssenteret.no/publikasjoner/ attachment/4128?_ts=11d1a7a8760\&download=false. Accessed 22 Mar 2014.

39. The Norwegian Directorate of Health. Economic evaluation of health interventions-a guideline [in Norwegian]. http://www. helsedirektoratet.no/publikasjoner/okonomisk-evaluering-av-hel etiltak-en-veileder/Publikasjoner/IS-1985.pdf (2012 IS-1985). Accessed 22 Mar 2014

40. Lunde L. Economic evaluations in the health care market: methodological issues and challenges [PhD thesis]. Bergen: University of Bergen; 2012.

41. Berg J, Lindgren P, Nieuwlaat R, Bouin O, Crijns H. Factors determining utility measured with the EQ-5D in patients with atrial fibrillation. Qual Life Res. 2010;19(3):381-90.

42. Leontiadis GI, Sreedharan A, Dorward S, Barton P, Delaney B, Howden CW, et al. Systematic reviews of the clinical effectiveness and cost-effectiveness of proton pump inhibitors in acute upper gastrointestinal bleeding. Health Technol Assess. 2007; 11(51): iii-iv, 1-164.

43. Lee HY, Hwang JS, Jeng JS, Wang JD. Quality-adjusted life expectancy (QALE) and loss of QALE for patients with ischemic stroke and intracerebral hemorrhage: a 13-year follow-up. Stroke. 2010;41(4):739-44.

44. Coleman CI, Sobieraj DM, Winkler S, Cutting P, Mediouni M, Alikhanov S, et al. Effect of pharmacological therapies for stroke prevention on major gastrointestinal bleeding in patients with atrial fibrillation. Int J Clin Pract. 2012;66(1):53-63.

45. Rasanen P, Roine E, Sintonen H, Semberg-Konttinen V, Ryynanen OP, Roine R. Use of quality-adjusted life years for the estimation of effectiveness of health care: a systematic literature review. Int J Technol Assess Health Care. 2006;22(2):235-41.

46. Wisløff T, Hagen G, Hamidi V, Movik E, Klemp M, Olsen JA. Estimating QALY gains in applied studies: a review of costutility analyses published in 2010. Pharmacoeconomics. Epub 2014 Jan 30. doi:10.1007/s40273-014-0136-z.

47. Briggs AH, Claxton K, Sculpher M. Decision modelling for health economic evaluation. Oxford: Oxford University Press; 2006.

48. Altman DG. Practical statistics for medical research. 1st ed. London: Chapman \& Hall; 1991.

49. Thompson SG, Nixon RM. How sensitive are cost-effectiveness analyses to choice of parametric distributions? Med Decis Making. 2005;25(4):416-23. 
50. Briggs AH, Claxton K, Sculpher M. Decision modelling for health economic evaluation. Oxford: Oxford University Press; 2006.

51. Lainscak M, Cleland JG, Lenzen MJ, Keber I, Goode K, Follath $\mathrm{F}$, et al. Nonpharmacologic measures and drug compliance in patients with heart failure: data from the EuroHeart Failure Survey. Am J Cardiol. 2007;99(6B):31D-7D.

52. Fenwick E, Claxton K, Sculpher M. Representing uncertainty: the role of cost-effectiveness acceptability curves. Health Econ. 2001;10(8):779-87.

53. Claxton K, Sculpher M, McCabe C, Briggs A, Akehurst R, Buxton $M$, et al. Probabilistic sensitivity analysis for NICE technology assessment: not an optional extra. Health Econ. 2005;14(4):339-47.

54. Al MJ. Cost-effectiveness acceptability curves revisited. Pharmacoeconomics. 2013;31(2):93-100.

55. Weinstein MC, O’Brien B, Hornberger J, Jackson J, Johannesson $\mathrm{M}$, McCabe $\mathrm{C}$, et al. Principles of good practice for decision analytic modeling in health-care evaluation: report of the ISPOR Task Force on Good Research Practices-Modeling Studies. Value Health. 2003;6(1):9-17.

56. Wallentin L, Yusuf S, Ezekowitz MD, Alings M, Flather M, Franzosi MG, et al. Efficacy and safety of dabigatran compared with warfarin at different levels of international normalised ratio control for stroke prevention in atrial fibrillation: an analysis of the RE-LY trial. Lancet. 2010;376(9745):975-83.

57. Graff-Iversen S, Gjesdal K, Jugessur A, Myrstad M, Nystad W, Selmer R, et al. Atrial fibrillation, physical activity and endurance training [in English, Norwegian]. Tidsskr Nor Laegeforen. 2012; 132(3):295-9.

58. Wisløff T, Ringerike T, Hagen G, Reikvam Å, Klemp M. Efficacy and cost-effectiveness of new oral anticoagulants compared to warfarin for the prevention of stroke in patients with atrial fibrillation. 2013;5:1-79. http://www.kunnskapssenteret.no/ Publikasjoner/_attachment/18119?_ts=13df823146a\&download= true. Accessed 22 Mar 2014. 